\title{
Biceps Box Configuration for Superior Capsule Reconstruction of the Glenohumeral Joint Decreases Superior Translation but Not to Native Levels in a Biomechanical Study
}

\author{
Patrick J. Denard, M.D., Seungbum Chae, M.D., Christen Chalmers, B.S., \\ Jae Hyuk Choi, B.S.E., Michelle H. McGarry, M.S., Gregory Adamson, M.D., \\ Max Park, M.D., and Thay Q. Lee, Ph.D.
}

\begin{abstract}
Purpose: To quantitatively biomechanically assess superior stability, subacromial contact pressures, and glenohumeral kinematics of an in situ biceps tenodesis and a box-shaped long head of the biceps tendon (LHBT) superior capsule reconstruction (SCR) in a superior massive rotator cuff tear (MCT) model. Methods: Eight cadaveric shoulders (mean age, 62 years; range, 46-70 years) were tested with a custom testing system used to evaluate range of motion, superior translation, and subacromial contact pressure at $0^{\circ}, 20^{\circ}$, and $40^{\circ}$ of abduction. Conditions tested included native state, MCT (complete supraspinatus and one-half of the infraspinatus), a box-shaped LHBT SCR, and an in situ biceps tenodesis. The box-shaped SCR was performed by maintaining the biceps origin, securing 2 corners to the greater tuberosity, and one corner to the posterior glenoid. The in situ tenodesis was performed anatomically at the top of the articular margin in the same shoulder after take-down of the box SCR. Results: Range of motion was not impaired with either repair construct $(P>.05)$. The box SCR decreased superior translation by approximately $2 \mathrm{~mm}$ compared with the MCT at $0^{\circ}$, but translation remained greater compared with the intact state in nearly every testing position. The in situ tenodesis had no effect on superior translation. Peak subacromial contact pressure was increased in the MCT at $0^{\circ}$ and $20^{\circ}$ abduction compared with the native state but not different between the native and box SCR at the same positions. Conclusions: A box-shaped SCR using the native biceps tendon partially restores increased superior translation and peak subacromial contact pressure due to МСT. The technique may have a role in augmentation of an irreparable MCT. Clinical Relevance: The box-shaped LHBT SCR technique may have a role in augmentation of an irreparable MCT.
\end{abstract}

$\mathbf{T}$ he superior capsule of the glenohumeral joint is a distinct anatomical structure that plays a vital biomechanical role in the shoulder. ${ }^{1,2}$ In the setting of a massive irreparable rotator cuff repair, stability of the glenohumeral joint is disrupted. An absent superior capsule allows for passive superior translation and hence an

From Oregon Shoulder Institute, Medford, Oregon, U.S.A. (P.J.D.); Orthopaedic Biomechanics Laboratory, Congress Medical Foundation, Pasadena, California, U.S.A. (S.C., C.C., J.H.C., M.H.M., G.A., T.Q.L.); Department of Orthopaedic Surgery, Daegu Catholic University Hospital School of Medicine, Daegu, Korea (S.C); and Southern California Permanente Group, Woodland Hills, California, U.S.A. (M.P.).

The authors report the following potential conflicts of interest or sources of funding: M.P. reports grants from Arthrex, during the conduct of the study. In addition, he has a patent relating to the biceps tendon as a graft issued. T.Q.L. reports grants and nonfinancial support from Arthrex, during the conduct of the study, and personal fees from Arthrex, outside the submitted work. In addition, he has a patent relating to the biceps tendon as a graft issued. P.J.D. reports grants from Arthrex, during the conduct of the study, and personal fees impaired fulcrum for glenohumeral abduction. Superior capsule reconstruction (SCR) has been shown biomechanically to restore superior stability and is supported with clinical outcomes up to 5 years after surgery. ${ }^{3,4}$

A variety of graft sources have been proposed for SCR. Initially, Mihata et al. ${ }^{5}$ reported the use of tensor

and other from Arthrex, outside the submitted work. Full ICMJE author disclosure forms are available for this article online, as supplementary material.

Received May 15, 2020; accepted September 22, 2020.

Address correspondence to Patrick J. Denard, M.D., 2780 E. Barnett Rd., Suite 200, Medford, OR 97504.E-mail:pjdenard@gmail.com

(C) 2020 THE AUTHORS. Published by Elsevier Inc. on behalf of the Arthroscopy Association of North America. This is an open access article under the CC BY-NC-ND license (http://creativecommons.org/licenses/by-nc-nd/4.0/). 2666-061X/20717

https://doi.org/10.1016/j.asmr.2020.09.024 
fascia lata (TFL) autograft. While supported with clinical outcomes, graft harvest is associated with increased donor-site morbidity and is difficult to perform if the patient is positioned in beach chair. Dermal allograft has been used extensively for SCR because it addresses these limitations well. ${ }^{6}$ However, dermal allograft is costly and cannot be used in some countries, which restrict the use of allografts. Moreover, while functional outcomes have been encouraging, healing of dermal allograft has been variable. ${ }^{7-10}$ Another alternative is the use of the long head of the biceps tendon (LHBT). The LHBT has a history of use in augmentation of rotator cuff repairs ${ }^{11}$ and recently has been described for SCR. ${ }^{12,13}$ A variety of graft configurations have been described, but biomechanical evaluation of the constructs has been limited. ${ }^{14,15}$

The purpose of this study was to quantitatively biomechanically assess superior stability, subacromial contact pressures, and glenohumeral kinematics of an in situ biceps tenodesis and a box-shaped LHBT SCR in a massive rotator cuff tear model. The hypothesis of this study was that both the in situ biceps tenodesis and box-shaped LHBT SCR would restore superior translation to the intact state following creation of a massive rotator cuff tear (MCT).

\section{Methods}

\section{Specimen Preparation}

Eight fresh frozen cadaveric shoulders from 2 female and 6 male donors (mean age, 62 years; range, 46-70 years) were tested using a previously described custom shoulder testing system. Specimens were dissected, removing all skin and subcutaneous tissue but preserving the rotator cuff, biceps, pectoralis major, latissimus dorsi, deltoid, and coracoacromial ligament. The humerus was transected $2 \mathrm{~cm}$ distal to the deltoid insertion. None of the donors showed any evidence of rotator cuff tendon, superior labrum, or proximal biceps tendon tears.

\section{Setup}

The scapula was mounted to a custom shoulder testing system with the scapula placed in $0^{\circ}$ abduction and $20^{\circ}$ anterior tilt in the sagittal plane (Fig 1). The humeral shaft was secured with an intramedullary rod, which allowed control of axial rotation and glenohumeral abduction. A digital goniometer (Novotechnik US, Southborough, MA) was placed at the distal end of the humerus to measure range of motion. Ninety degrees of external rotation was defined as the position at which the anterior edge of the acromion aligned with the LHBT in the bicipital groove at $60^{\circ}$ of glenohumeral abduction. Zero glenohumeral abduction was defined as the position at which the humeral rod lined up perpendicular to the ground.

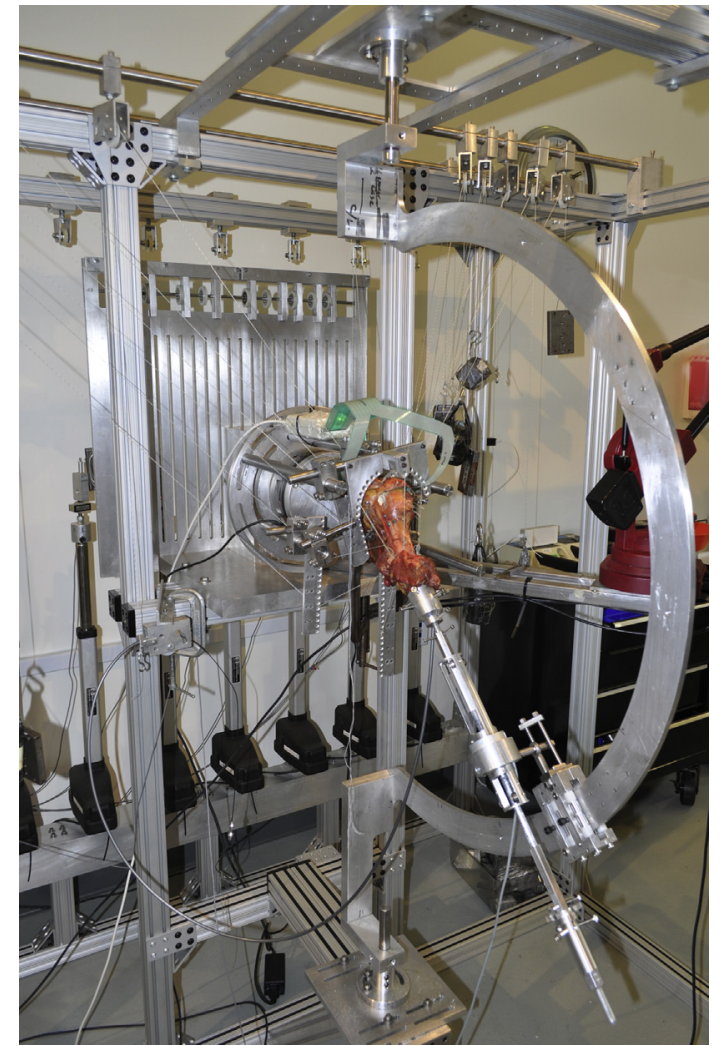

Fig 1. Photograph of the custom shoulder testing system with a left shoulder specimen in $40^{\circ}$ of glenohumeral abduction.

A muscle loading ratio based on physiological crosssectional area of the muscle was used as previously described (Fig 2). ${ }^{15}$ Each testing condition was performed using 2 different loading conditions: balanced load (deltoid, 40N; pectoralis major, 20N; latissimus dorsi, 20N; supraspinatus, $5 \mathrm{~N}$; subscapularis, $10 \mathrm{~N}$; infraspinatus, $5 \mathrm{~N}$; teres minor, $5 \mathrm{~N}$; biceps, $2.5 \mathrm{~N}$ ) and a superiorly directed load (deltoid, 80N; supraspinatus, $5 \mathrm{~N}$; subscapularis, $10 \mathrm{~N}$; infraspinatus, $5 \mathrm{~N}$; teres minor, $5 \mathrm{~N}$; biceps, $2.5 \mathrm{~N}$ ). The supraspinatus and anterior half of infraspinatus were unloaded for all conditions following the simulated irreparable cuff tear. A $5-\mathrm{N}$ load was applied to the biceps tendon in the intact state and MCT model. This load was removed for the LHBT box SCR and then reapplied for the in situ tenodesis.

\section{Measurements}

The humeral rod was inserted through a hollow-shaft angle pontentiometer (Novotechnik US) for measuring humeral rotation. The potentiometer was attached to an arc with a custom device that allows for compression/distraction, anterior/posterior, and medial/lateral glenohumeral translation while fixing the degree of shoulder abduction. Humeral rotational range of motion was measured in the balanced condition with an 


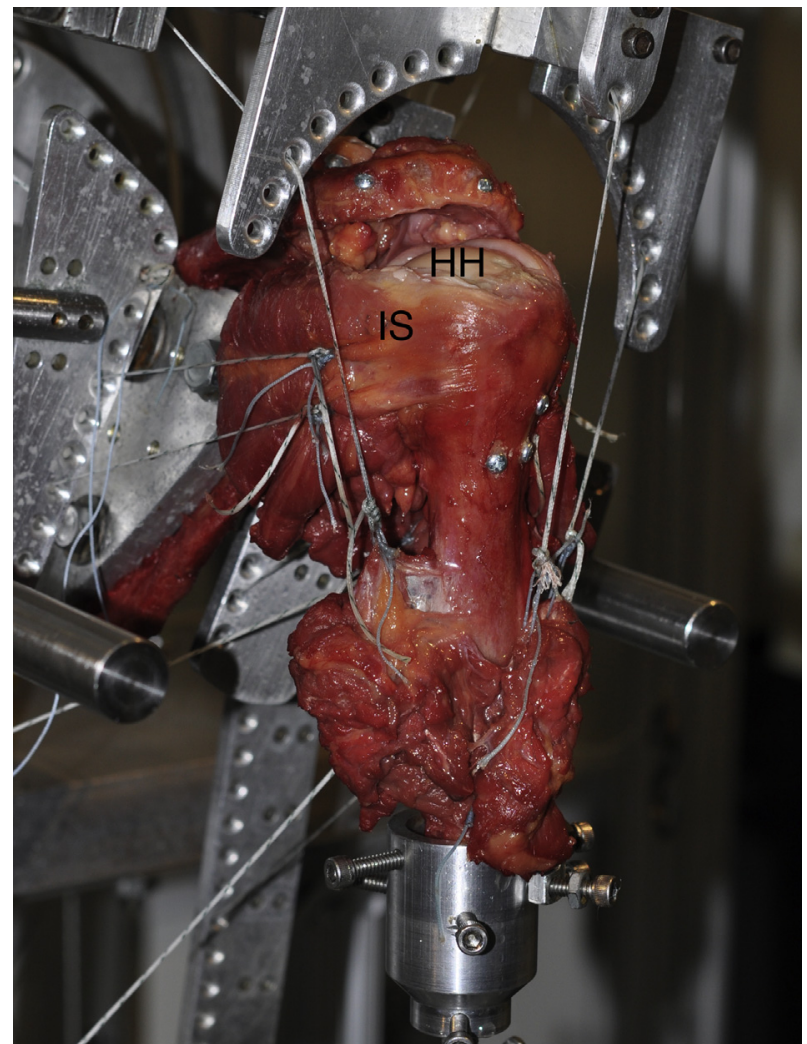

Fig 2. Photograph of a right shoulder following creation of a massive rotator cuff tear showing the muscle loading of each individual tendon. The infraspinatus (IS) is to left. The supraspinatus tendon has been removed revealing the superior aspect of the humeral head $(\mathrm{HH})$.

application of $2.2 \mathrm{Nm}$ of torque to the humerus in the internal and external directions. Specimens were preconditioned with 5 cycles before measurements.

Positional change of the humeral head relative to the glenoid was evaluated in both balanced and unbalanced conditions. Three markers each were placed on the scapula and proximal humerus and digitized with a Microscribe 3DLX (Revware, Raleigh, NC). which has an accuracy within $<0.3 \mathrm{~mm}$. Superior translation was calculated as the difference in humeral head position from the unbalanced to balanced loading condition.

Subacromial contact pressure was evaluated in the unbalanced loading condition. A Tekscan pressure sensor (Model 4000; maximum pressure 10.3 MPa; Tekscan, South Boston, MA) was placed in the subacromial space and used to measure contact force, contact area, contact pressure, and peak contact pressure between the undersurface of the acromion and the greater tuberosity. The sensor's sensitivity was set to 35 $\mathrm{N}$ and calibrated using a 2-point calibration protocol with an applied force of $40 \mathrm{~N}$ and $80 \mathrm{~N}$ using an Instron 4411 load cell (Instron, Norwood, MA).

All measurements were performed at $0^{\circ}, 20^{\circ}$, and $40^{\circ}$ of glenohumeral abduction (equivalent to $0^{\circ}, 30^{\circ}, 60^{\circ}$ of shoulder abduction) and $0^{\circ}, 30^{\circ}, 60^{\circ}, 90^{\circ}$, of humeral rotation.

\section{Tested Conditions}

Four conditions were tested: the intact state, a stage III MCT model (complete supraspinatus and anterior one-half of the infraspinatus), a box-shaped SCR using the LHBT (Box Biceps SCR), and a single-limb in situ biceps tenodesis (single-limb biceps). In the MCT model, the capsule and rotator cuff were resected medially and no load was applied to the supraspinatus or anterior one-half of the infraspinatus.

All reconstructions were performed by one author (S.C.). The Box Biceps SCR is based on 4 points of fixation in a box shape designed to mimic the normal origin and attachment of the superior capsule. ${ }^{16}$ The anteromedial fixation is that of the native LHBT origin, whereas the other 3 corners are created with 3 knotless anchors. The procedure was performed with the arm in $20^{\circ}$ of abduction and $30^{\circ}$ of external rotation. ${ }^{15}$ The native origin of the LHBT was left intact. First, the LHBT was tenodesed at the top of the bicipital groove, just lateral to the articular margin with a $4.75-\mathrm{mm}$ knotless anchor (BioComposite SwiveLock C; Arthrex, Naples, FL) using a \#2 suture through the graft in a "Loop ' $n$ ' Tack" technique. ${ }^{17}$ This created the anterolateral corner of the box. Next, the bicipital groove was opened and the distal aspect of the tendon was transected just above the musculotendinous junction. The distal tendon was whip-stitched with a \#2 suture for $2 \mathrm{~cm}$ and then secured to the glenoid approximately $12 \mathrm{~mm}$ posterior to the native biceps anchor with another $4.75-\mathrm{mm}$ knotless anchor to create the posteromedial corner of the box. The last corner (posterolateral) of the box was created by securing the LHBT to the greater tuberosity footprint. A \#2 suture with a closed loop was passed around (vs through) the tendon to create a cinch suture. Then the tendon was secured to humerus with a third and final knotless anchor placed just anterior the margin of the remaining infraspinatus tissue. While the goal was to place the anchor at the articular margin, there was slight variance of medial to lateral position based on manual assessment of tension (no slack redundancy to the medial graft). Finally, 3 posterior side-to-side sutures were performed to attach the posterior limb of the Box Biceps SCR to the intact inferior half of the infraspinatus capsule and tendon. The sutures were passed around the biceps tendon and through the infraspinatus capsule (medial) and tendon (lateral) and then tied with a 6-throw surgeons knot. The sutures were deliberately passed around the biceps tendon as opposed to through the tendon in order to provide "forgiving" fixation that allows adaptation during internal and external rotation of the humerus (Fig 3). 

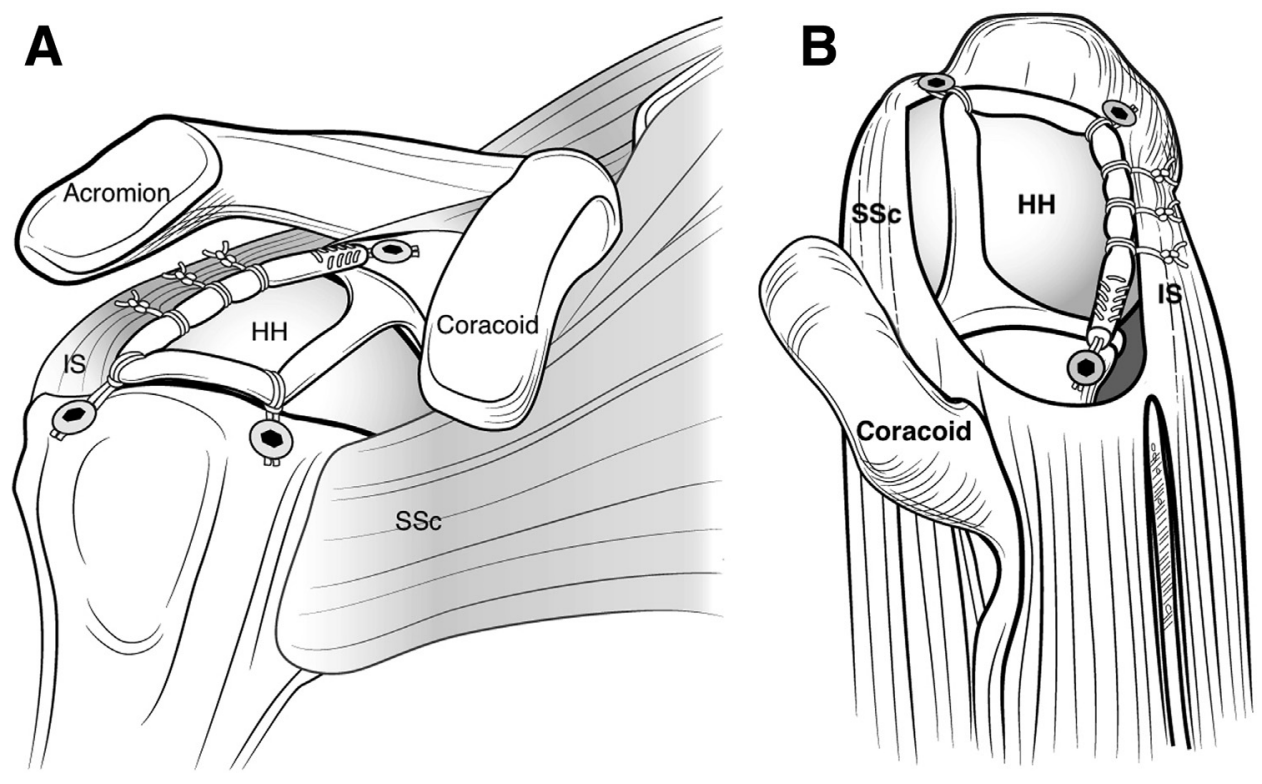

Fig 3. Illustration of the box biceps superior capsule reconstruction. The biceps origin is left intact. Anterolaterally, the biceps is secured at the top of the articular groove. Posterolaterally, the biceps is secured just anterior to the infraspinatus tendon insertion. Posteromedially the biceps is secured to the glenoid. ( $\mathrm{HH}$, humeral head; IS, infraspinatus; SSc, subscapularis.)
For the single-limb biceps condition, the posterior glenoid, posterior humeral head, and anterior humerus anchors were removed and the biceps tendon was placed back in the bicipital groove so that a load could be applied to the biceps for testing. The biceps was then tenodesed again with a "Loop ' $\mathrm{n}$ ' Tack" configuration using a 5.5-mm SwiveLock anchor in the same anterior location with the same glenohumeral position as for the Box Biceps SCR (Fig 4).

\section{Statistical Analysis}

The primary outcome of interest was superior translation. The secondary outcomes of interest were subacromial contact pressure and glenohumeral range of motion. A 2-way repeated-measures analysis of variance was used for statistical analysis to compare between the four testing conditions. If a significant difference was detected pairwise comparisons were performed with a Bonferroni correction to determine where the significant differences were. Data are presented as means \pm standard error of the mean and the significance level was set at $P<.05$.

\section{Results}

\section{Range of Motion}

Relative to intact, the simulated massive cuff tear significantly increased internal rotation at $40^{\circ}$ abduction $(P=.003)$ and total range of motion at $0^{\circ}(P=$ $.049)$ and $40^{\circ}$ abduction $(P=.003)$. Neither the Box Biceps SCR nor the in situ single limb tenodesis resulted in reductions of range of motion compared to the intact or simulated rotator cuff tear states. There were no significant differences in range of motion between the single limb biceps and Box Biceps SCR at any abduction angles (Table 1).

\section{Superior Translation}

At $0^{\circ}$ abduction, the MCT significantly increased superior translation of the humeral head at all degrees of humeral rotation relative to intact (Fig 5A). Following the Box Biceps SCR, superior translation of the humeral head was significantly decreased relative to the MCT at all degrees of humeral rotation $\left(0^{\circ}, P=.019 ; 30^{\circ}, P<\right.$ $\left.0.001 ; 60^{\circ}, P=.006 ; 90^{\circ}, P=.006\right)$ but remained increased compared to the intact state at $0^{\circ}, 30^{\circ}$, and $60^{\circ}$ of external rotation. The single-limb biceps did not result in reduction of superior translation compared to the MCT state.

At $20^{\circ}$ abduction, superior translation was significantly increased compared to the native state in all degrees of humeral rotation (Fig 5B). The Box Biceps SCR significantly decreased superior translation of the humeral head relative to the MCT at $0^{\circ}$ external rotation only $(P=.039)$. The single-limb biceps did not significantly reduce superior translation relative to the MCT at any rotation $(P>.095)$.

At $40^{\circ}$ abduction, superior translation was increased in the MCT compared with the intact state at $30^{\circ}(P=$ $.021)$ and $60^{\circ}$ of external rotation $(P=.026)$ (Fig $5 \mathrm{C}$ ). Following the Box Biceps SCR, superior translation of the humeral head remained significantly increased relative to intact at all external rotation angles $\left(0^{\circ}, P=\right.$ $\left..012 ; 30^{\circ}, P=.013 ; 60^{\circ}, P=.015 ; 90^{\circ}, P=.001\right)$. Following the single-limb biceps superior translation remained significantly increased compared to intact at $0^{\circ}(P=.043), 60^{\circ},(P=.035)$, and $90^{\circ}$ of external rotation $(P=.020)$. There were no significant 


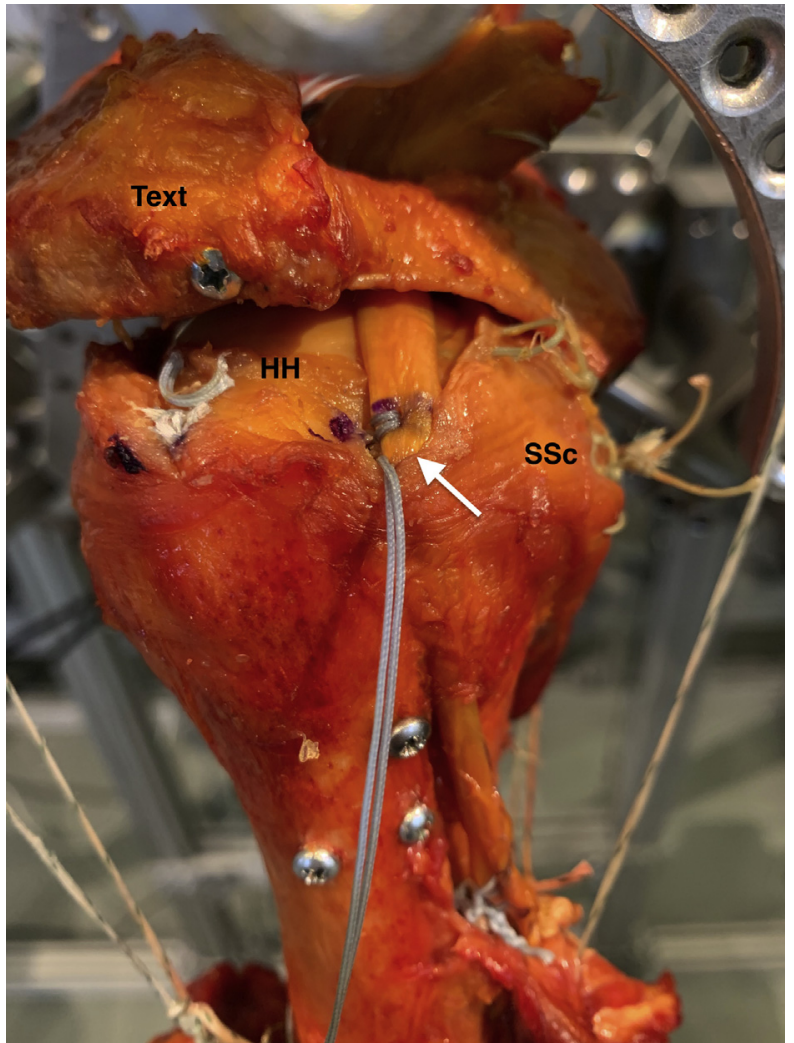

Fig 4. Photograph of a right shoulder demonstrating the in situ single-limb biceps tenodesis (white arrow). The biceps has been tenodesed along its normal course at the top of the articular margin of the humeral head (HH). (SSc, subscapularis.)

differences between the massive cuff tear and both the Box Biceps SCR and single limb biceps or between the Box Biceps SCR and single limb biceps at any of the rotation angles $(P>.05$ for all comparisons).

\section{Subacromial Contact Pressure}

At $0^{\circ}$ abduction, peak subacromial contact pressure was increased in the MCT model relative to intact at $60^{\circ}$ external rotation $(P=.006)$ (Fig 6A). Following Box Biceps SCR, peak contact pressure at this position was decreased relative to the MCT model $(P=.026)$ and not significantly greater that the native state $(P=.999)$.

At $20^{\circ}$ abduction and $30^{\circ}$ and $60^{\circ}$ external rotation, peak subacromial contact pressure was increased relative to intact following both the MCT $(P=.014, P=$ .019 , respectively) and single-limb biceps $(P=.014, P=$ .008 , respectively) (Fig 6B).

At $40^{\circ}$ abduction, peak subacromial contact pressure was significantly increased compared with intact following all 3 testing conditions at $60^{\circ}$ external rotation (vs MCT $P=.020$; vs box biceps $P=.022$; vs singlelimb biceps $P=.026$ ) (Fig 6C).

\section{Discussion}

The major findings of this study were that superior translation following a MCT was reduced with a boxshaped SCR using the native biceps tendon but could not be reduced following a single limb in situ biceps tenodesis. Contrary to the study hypothesis, superior translation following a MCT was not restored to the intact state following either a box-shaped SCR or in-situ biceps tenodesis. However, the Box Biceps SCR significantly reduced superior translation compared to the MCT condition, demonstrating potential clinical utility.

Glenohumeral joint stability is provided by a combination of dynamic (i.e., rotator cuff and LHBT) and static (i.e., superior capsule) stabilizers. In the setting of a massive irreparable rotator cuff tear the superior capsule is disrupted, leading to decreased resistance to superior translation. ${ }^{2}$ SCR has emerged as a technique for restoring biomechanical stability of the glenohumeral joint. Mihata et al. ${ }^{4}$ demonstrated that SCR with TFL is capable of restoring superior translation to the native state. The biomechanical findings of this study were corroborated by clinical studies demonstrating restoration of the acromiohumeral distance and improvement in functional outcomes with SCR

Table 1. Humeral Rotational Range of Motion for Each Abduction Angle and Conditions

\begin{tabular}{|c|c|c|c|c|}
\hline & Intact & MCR & Box Biceps Superior Capsule Reconstruction & In Situ Single-Limb Tenodesis \\
\hline \multicolumn{5}{|l|}{ Internal rotation, $^{\circ}$} \\
\hline $0^{\circ}$ abduction & $13.6 \pm 3.4$ & $17.1 \pm 2.9$ & $16.9 \pm 3.7$ & $18.6 \pm 4.0$ \\
\hline $20^{\circ}$ abduction & $21.2 \pm 2.8$ & $23.1 \pm 2.7$ & $23.5 \pm 3.5$ & $23.8 \pm 3.5$ \\
\hline $40^{\circ}$ abduction & $21.3 \pm 3.1$ & $24.1 \pm 3.3 *$ & $24.9 \pm 3.7^{*}$ & $25.3 \pm 3.9$ \\
\hline \multicolumn{5}{|l|}{ External rotation, ${ }^{\circ}$} \\
\hline $0^{\circ}$ abduction & $104.0 \pm 3.6$ & $106.7 \pm 3.7$ & $108.1 \pm 3.6^{* \dagger}$ & $107.7 \pm 3.6^{* \dagger}$ \\
\hline $20^{\circ}$ abduction & $118.4 \pm 2.4$ & $118.6 \pm 2.6$ & $119.7 \pm 2.5$ & $118.8 \pm 2.7$ \\
\hline $40^{\circ}$ abduction & $120.6 \pm 2.2$ & $121.4 \pm 2.4$ & $123.4 \pm 2.6$ & $122.8 \pm 2.5$ \\
\hline \multicolumn{5}{|l|}{ Total rotation, $^{\circ}$} \\
\hline $0^{\circ}$ abduction & $117.6 \pm 6.6$ & $123.8 \pm 6.4^{*}$ & $125.1 \pm 7.1$ & $126.3 \pm 7.4$ \\
\hline $20^{\circ}$ abduction & $139.6 \pm 4.6$ & $141.7 \pm 4.7$ & $143.2 \pm 5.5$ & $142.6 \pm 5.7$ \\
\hline $40^{\circ}$ abduction & $141.9 \pm 4.3$ & $145.5 \pm 4.6^{*}$ & $148.4 \pm 5.4^{*}$ & $148.1 \pm 5.5^{*}$ \\
\hline
\end{tabular}

NOTE. Data are presented as mean \pm standard error of the mean. $(P<.05 *$ vs intact; †vs MCT $)$.

MCT, massive cuff tear. 

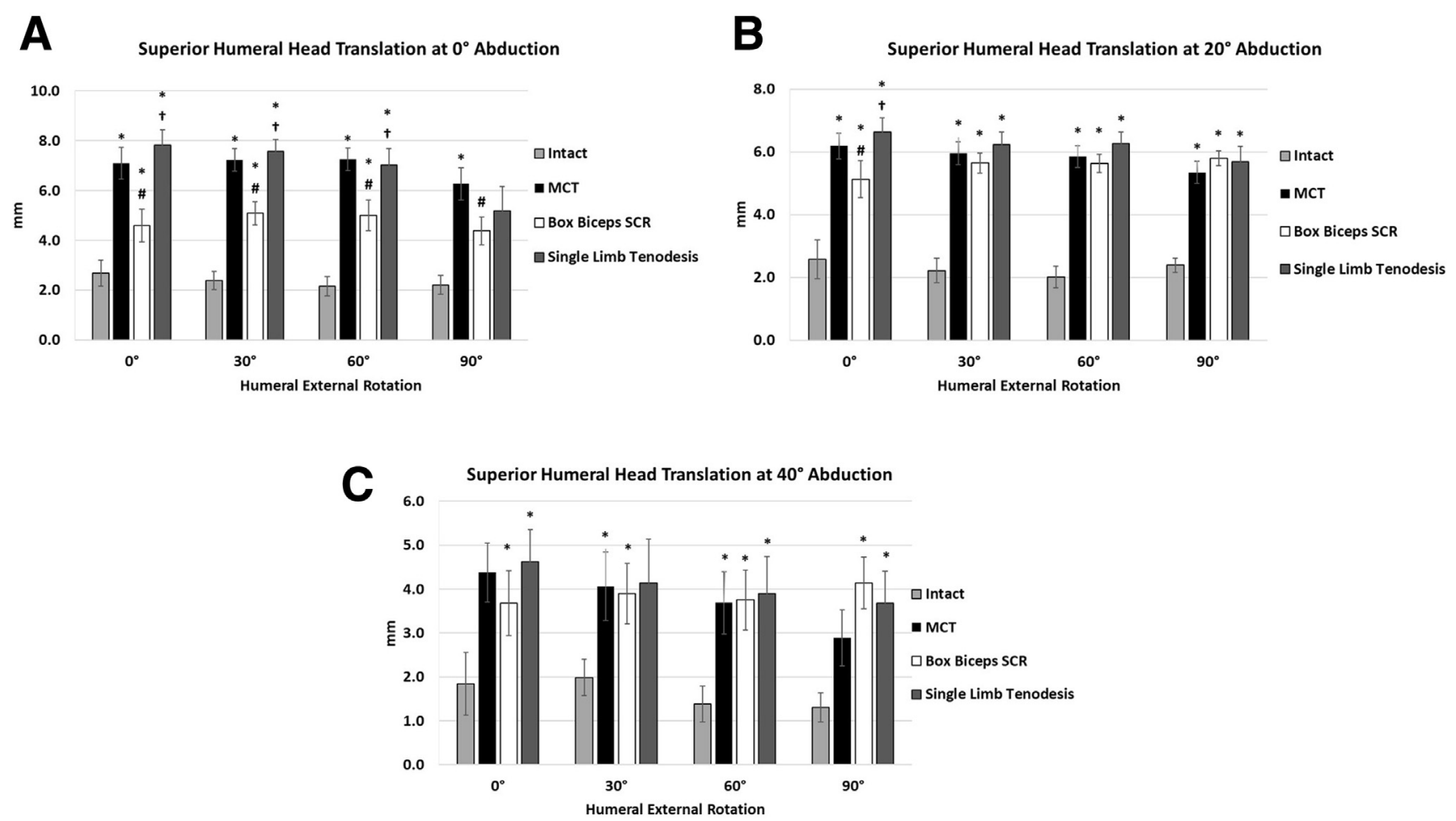

Fig 5. Amount of superior translation (millimeters) from unbalanced to balanced muscle load for each humeral rotation position and specimen condition. (A) $0^{\circ}$ glenohumeral abuction; (B) $20^{\circ}$ glenohumeral abuction; and (C) $40^{\circ}$ glenohumeral abuction. $\left(P<.05 ;{ }^{*}\right.$ vs intact, \#vs massive cuff tear [MCT] and †vs box biceps superior capsule reconstruction [SCR]).

performed with autograft TFL. ${ }^{3,5}$ Nonetheless, other authors have sought alternatives to TFL to avoid donorsite morbidity as well as the fact that TFL is difficult to harvest in the beach chair position during arthroscopic surgery. ${ }^{6,8}$

Recently, the LHBT has been proposed as an alternative graft source for partial reconstruction of the superior capsule because it is low cost and avoids the donor-site morbidity associated with harvesting TFL. ${ }^{12,13,18}$ It also can be harvested from either the lateral decubitus or beach-chair position. In a biomechanical study, El-Shaar et al. ${ }^{19}$ compared SCR with either the LHBT or TFL. For the biceps SCR, the origin was left intact and the tendon was cut distally and whipstitched so that it could be re-routed back into the joint. The distal end was secured to the posterior glenoid with 2.9-mm knotless push-in anchor and the middle portion was fixed to the middle of the greater tuberosity with an 8-mm knotless threaded anchor to create a " $V$ " configuration. The LHBT SCR required $393 \%$ increased force for superior translation compared with a MCT state and the TFL required 194\% increased force, with a trend toward a greater force required in the TLF compared with the LHBT $(P=.059)$. The authors concluded that the LHBT was equivalent to and "potentially even stronger" than TFL SCR, but did not report range of motion, superior translation, or subacromial contact pressure.
In the current study, superior translation was reduced with the box LHBT SCR but remained greater that than of the native state. At $0^{\circ}$ of abduction and $30^{\circ}$ of external rotation, for instance, superior translation was $2.4 \mathrm{~mm}$ at baseline, increased to $7.2 \mathrm{~mm}$ following the MCT model, and decreased to $5.1 \mathrm{~mm}$ with the LHBT SCR. This represented a statistically significant $29 \%$ reduction compared with the $\mathrm{MCT}$, but the translation still remained more than $200 \%$ greater than the intact state. Notably, there were few differences in peak subacromial contact pressure between the native state and Box LHBT SCR, suggesting the latter is capable of providing a spacer effect. Given this spacer effect and the partial reduction in superior translation, it is possible that the biceps Box SCR is an option for augmentation of a repair or an alternative for a less severe RCT such as an irreparable stage II tear (supraspinatus alone).

Ultimately, the technical difficulty of the box SCR configuration and weaknesses of the fixation with our technique should be weighed against simpler options using the biceps tendon. Park et al. performed a biomechanical investigation of the biceps tendon for anterior cable reconstruction alone in the same MCT model used in the current study. ${ }^{15}$ The biceps was secured anterior to the given MCT condition with a double-row repair to optimize healing surface area and then sutured to the posterior infraspinatus with 

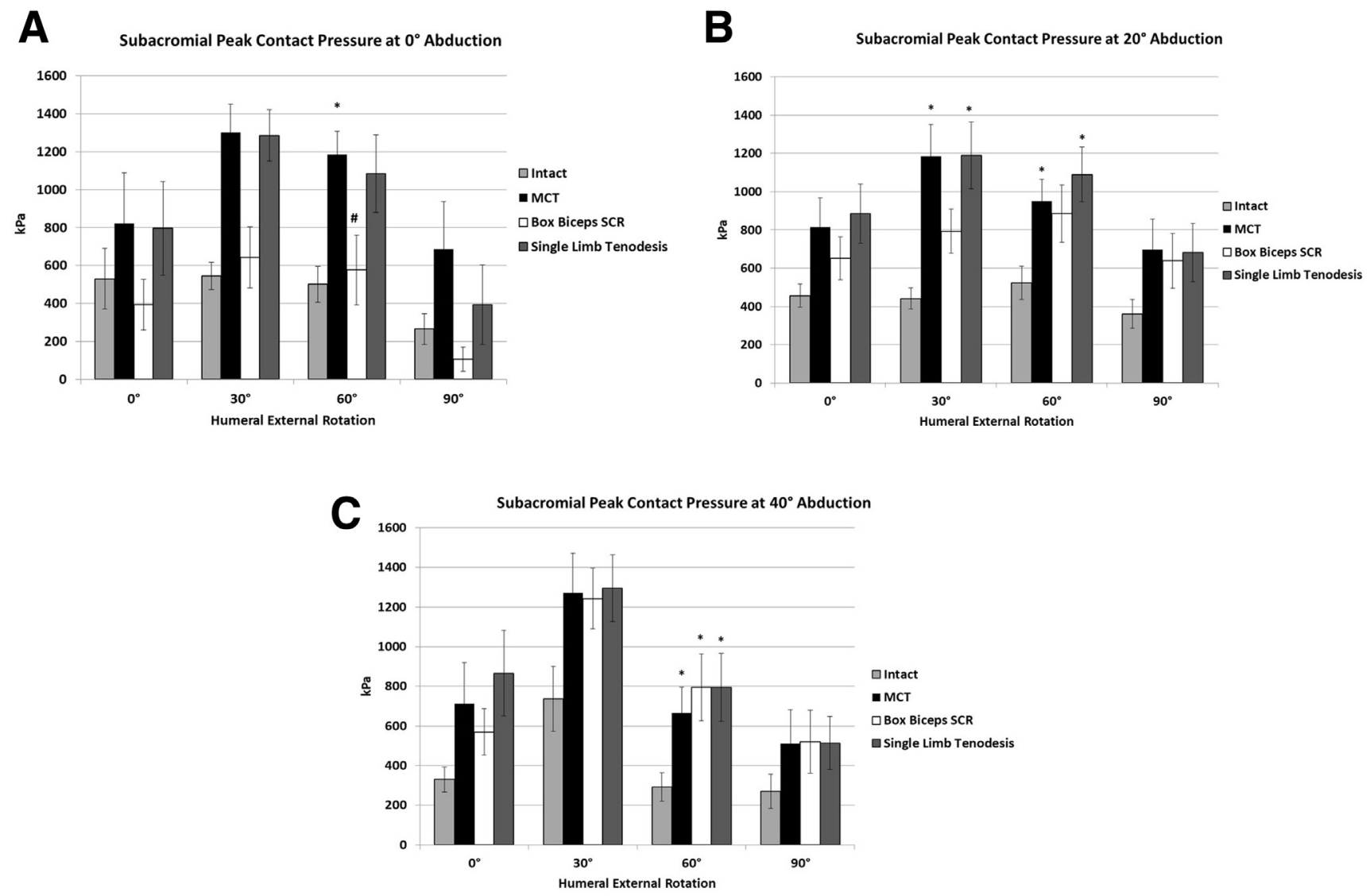

Fig 6. Subacromial peak contact pressure with unbalanced muscle load for each humeral rotation position and specimen condition. (A) $0^{\circ}$ glenohumeral abuction; (B) $20^{\circ}$ glenohumeral abuction; and (C) $40^{\circ}$ glenohumeral abuction. $(P<.05$; *vs intact, \#vs massive cuff tear [MCT] and †vs box biceps superior capsule reconstruction [SCR]).

side-to-side sutures. Remarkably, this construct restored superior translation to native levels. This difference compared with our findings may be explained by the enhanced fixation in their study. Whereas they used a double-row technique, the corners of the biceps in our configuration provided single-point fixation. Thus, while having multiple limbs cross the biomechanical joint may have some biologic advantage, from a biomechanical perspective, strength of fixation is likely more important.

In the native state, when placed under load, the LHBT provides a stabilizing effect against superior translation of the humeral head. ${ }^{20}$ Unlike the static stabilization of the superior capsule, the LHBT crosses the glenohumeral joint and extends distally. There is thus concern that in situ tenodesis without resection of the tendon distally may limit glenohumeral ROM. In the current study the in situ tenodesis did not significantly lower glenohumeral motion compared with either the native or MCT states, suggesting that an in situ tenodesis is safe from the perspective of ROM. The in-situ tenodesis, however, provided only minimal resistance to superior translation with only a slight reduction in superior translation at $0^{\circ}$ of abduction and $60^{\circ}$ of external rotation compared with the MCT state. Therefore, the in situ tenodesis cannot be recommended alone as a solution for an irreparable MCT. In contrast, it is possible that in situ tenodesis could be used to supplement a SCR performed with another graft source.

One question to consider is if there is adequate length of the biceps tendon to reliably be used in a box configuration. Denard et al. ${ }^{21}$ previously investigated the normal anatomic relationships of the biceps in a cadaveric study. They reported that the mean length of the biceps tendon from the origin to the musculotendinous junction was $98.5 \mathrm{~mm}$. The distance from the origin to the articular margin of the humeral head was $24.9 \mathrm{~mm}$. Assuming an anterior-posterior distance for the greater tuberosity of $20-25 \mathrm{~mm}$ and then an additional $25-30 \mathrm{~mm}$ of graft length to route back to the glenoid, the average graft length required is $70-80 \mathrm{~mm}$, which is less than the graft length that should be available.

\section{Limitations}

There are several limitations to our study. The findings are limited to the biomechanical analysis and do 
reflect biologic healing. We did not compare the box SCR construct with another graft source for SCR such as TFL. There are also several disadvantages of the box SCR technique. First, in the native state the biceps tendon contributes to superior stability of the glenohumeral joint as noted previously. Thus, sacrificing the biceps tendon for a box configuration SCR may be a trade-off. It is notable that in the initial technique description of Mihata et al. ${ }^{5}$ left the biceps tendon in place as opposed to performing tenotomy or tendoesis. Second, the biceps tendon is not always present in the setting of a massive irreparable rotator cuff tear. Third, the technique is still somewhat challenging. It requires a separate incision for harvest of the biceps distally. In this study, we performed the repair in an open manner which made management of the graft relatively simple. However, graft management would be more difficult arthroscopically.

\section{Conclusions}

A box-shaped SCR using the native biceps tendon partially restores increased superior translation and peak subacromial contact pressure due to MCT. The technique may have a role in augmentation of an irreparable MCT.

\section{References}

1. Adams CR, DeMartino AM, Rego G, Denard PJ, Burkhart SS. The Rotator cuff and the superior capsule: Why we need both. Arthroscopy 2016;32:2628-2637.

2. Ishihara Y, Mihata T, Tamboli M, et al. Role of the superior shoulder capsule in passive stability of the glenohumeral joint. J Shoulder Elbow Surg 2014;23:642-648.

3. Mihata T, Lee TQ, Hasegawa A, et al. Five-year follow-up of arthroscopic superior capsule reconstruction for irreparable rotator cuff tears. J Bone Joint Surg Am 2019;101: 1921-1930.

4. Mihata T, McGarry MH, Pirolo JM, Kinoshita M, Lee TQ. Superior capsule reconstruction to restore superior stability in irreparable rotator cuff tears: A biomechanical cadaveric study. Am J Sports Med 2012;40:2248-2255.

5. Mihata T, Lee TQ, Watanabe C, Fukunishi K, Ohue M, Tsujimura T, et al. Clinical results of arthroscopic superior capsule reconstruction for irreparable rotator cuff tears. Arthroscopy 2013;29:459-470.

6. Burkhart SS, Denard PJ, Adams CR, Brady PC, Hartzler RU. Arthroscopic superior capsular reconstruction for massive irreparable rotator cuff repair. Arthrosc Tech 2016;5:e1407-e1418.

7. Burkhart SS, Pranckun JJ, Hartzler RU. Superior capsular reconstruction for the operatively irreparable rotator cuff tear: Clinical outcomes are maintained 2 years after surgery. Arthroscopy 2020;36:373-380.

8. Denard PJ, Brady PC, Adams CR, Tokish JM, Burkhart SS. Preliminary results of arthroscopic superior capsule reconstruction with dermal allograft. Arthroscopy 2018;34: 93-99.

9. Lacheta L, Horan MP, Schairer WW, et al. Clinical and imaging outcomes after arthroscopic superior capsule reconstruction with human dermal allograft for irreparable posterosuperior rotator cuff tears: A minimum two year follow up. Arthroscopy 2020;36:1011-1019.

10. Lacheta L, Millett PJ. Editorial Commentary: Superior Capsule reconstruction using dermal allograft in posterosuperior rotator cuff tears-do patients benefit and allografts heal? Arthroscopy 2020;36:381-382.

11. Rhee YG, Cho NS, Lim CT, Yi JW, Vishvanathan T. Bridging the gap in immobile massive rotator cuff tears: Augmentation using the tenotomized biceps. Am J Sports Med 2008;36:1511-1518.

12. Boutsiadis A, Chen S, Jiang C, Lenoir H, Delsol P, Barth J. Long head of the biceps as a suitable available local tissue autograft for superior capsular reconstruction: "The Chinese way. Arthrosc Tech 2017;6:e1559-e1566.

13. Kim D, Jang Y, Park J, On M. Arthroscopic superior capsular reconstruction with biceps autograft: Snake technique. Arthrosc Tech 2019;8:e1085-el092.

14. Han F, Kong CH, Hasan MY, Ramruttun AK, Kumar VP. Superior capsular reconstruction for irreparable supraspinatus tendon tears using the long head of biceps: A biomechanical study on cadavers. Orthop Traumatol Surg Res 2019;105:257-263.

15. Park MC, Itami Y, Lin CC, et al. Anterior cable reconstruction using the proximal biceps tendon for large rotator cuff defects limits superior migration and subacromial contact without inhibiting range of motion: A biomechanical analysis. Arthroscopy 2018;34:2590-2600.

16. Pouliart N, Somers K, Eid S, Gagey O. Variations in the superior capsuloligamentous complex and description of a new ligament. J Shoulder Elbow Surg 2007;16:821-836.

17. Duerr RA, Nye D, Paci JM, Akhavan S. Clinical evaluation of an arthroscopic knotless suprapectoral biceps tenodesis technique: Loop 'n' tack tenodesis. Orthop J Sports Med 2018;6:2325967118779786.

18. Chiang $\mathrm{CH}$, Shaw L, Chih WH, Yeh ML, Su WR. Arthroscopic rotator cuff repair combined with modified superior capsule reconstruction as reinforcement by the long head of the biceps. Arthrosc Tech 2019;8: e1223-e1231.

19. El-Shaar R, Soin S, Nicandri G, Maloney M, Voloshin I, Superior capsular reconstruction with a long head of the biceps tendon autograft: A cadaveric study. Orthop J Sports Med 2018;6:2325967118785365.

20. McGarry MH, Nguyen ML, Quigley RJ, Hanypsiak B, Gupta R, Lee TQ. The effect of long and short head biceps loading on glenohumeral joint rotational range of motion and humeral head position. Knee Surg Sports Traumatol Arthrosc 2016;24:1979-1987.

21. Denard PJ, Dai X, Hanypsiak BT, Burkhart SS. Anatomy of the biceps tendon: Implications for restoring physiological length-tension relation during biceps tenodesis with interference screw fixation. Arthroscopy 2012;28: 1352-1358. 